\title{
Programas televisivos preferidos por los adolescentes de Lima y sus representaciones de violencia de pareja estereotipo de género y sexismo ambivalente
}

\author{
Television programs preferred by adolescents from Lima and its \\ representations of couple violence, gender stereotype and ambivalente \\ sexism
}

\author{
Alberto Quintana Peña ${ }^{1 *}$ \\ Alex Grajeda Montalvo ${ }^{2}$ \\ Carmela Malaver Soto ${ }^{3}$ \\ Nicolás Medina Curí ${ }^{4}$ \\ William Montgomery Urday ${ }^{5}$ \\ Universidad Nacional Mayor de San Marcos
}

\author{
Giancarlo Ojeda Mercado ${ }^{6}$ \\ Universidad Peruana Cayetano Heredia
}

Recibido: $07-10-17$

Aceptado: $21-11-17$

\section{Resumen}

Con el propósito de estudiar la diferencia entre las representaciones acerca de la violencia de pareja, estereotipos de género y sexismo ambivalente, se compara las representaciones de un grupo de adolescentes que prefieren ver telenovelas, con las representaciones de un grupo de adolescentes que prefieren ver realities de competencia. El Marco teórico de referencia será la teoría de las representaciones sociales de Moscovici y la de las redes semánticas referidas por Figueroa. Se evaluó a un grupo de 500 adolescentes, de ambos sexos, residentes en los conos norte, sur y este de Lima Metropolitana, usando como técnica de observación y análisis la Técnica de Redes Semánticas Naturales procesándose la data con el SPSS v.20, e interpretando las tablas y gráficos generados mediante la técnica de análisis de contenido. Finalmente, se concluye: que todos los integrantes de la muestra se representan la violencia como abuso físico y psicológico, que las adolescentes mujeres tienen una representación semánticamente más estable de los roles de género tradicional con predominio masculino, y enfatizan la belleza y el rol materno de la mujer.

Palabras clave: Representaciones; Violencia de pareja; Estereotipo de género; Sexismo ambivalente y Programas Televisivos.

\footnotetext{
1 Universidad Nacional Mayor de San Marcos. * Correspondencia, aquintanap@unmsm.edu.pe

2 Universidad Nacional Mayor de San Marcos. agrajedam@unmsm.edu.pe

3 Universidad Nacional Mayor de San Marcos. cmalavers@unmsm.edu.pe

4 Universidad Nacional Mayor de San Marcos.nmedinac@unmsm.edu.pe

5 Universidad Nacional Mayor de San Marcos. jmontgomeryu@unmsm.edu.pe

6 Universidad Peruana Cayetano Heredia. giancarlo.ojeda@upch.pe
}

(C) Los autores. Este artículo es publicado por la Revista de Investigación en Psicología de la Facultad de Psicología, Universidad Nacional Mayor de San Marcos. Este es un artículo de acceso abierto, distribuido bajo los términos de la licencia Creative Commons Atribucion - No Comercia_Compartir Igual 4.0 Internacional. (http://creativecommons.org/licenses/by-nc-sa/4.0/) que permite el uso no comercial, distribución y reproducción en cualquier medio, siempre que la obra original sea debidamente citada. 


\begin{abstract}
In order to study the difference between representations about partner violence, gender stereotypes and ambivalent sexism, we compare the representations of a group of adolescents who prefer to watch telenovelas, with the representations of a group of adolescents who prefer to see realities of competition. The theoretical frame of reference will be the theory of social representations of Moscovici and the theory of semantic networks referred by Figueroa. A group of 500 adolescents of both sexes, living in the northern and eastern cones of Metropolitan Lima, were evaluated using the Natural Semantic Networking Technique, processing the results with SPSS v.20, and interpreting tables and graphs generated by the technique of content analysis. Finally, it is concluded: that all the members of the sample represent violence as physical and psychological abuse, that female adolescents have a semantically more stable representation of traditional gender roles with male dominance, and adolescents emphasize beauty and parental role of women.
\end{abstract}

Keywords; Representations; Partner violence; Gender stereotype; Ambivalente sexism and Television programs.

Actualmente la Organización Mundial de la Salud reconoce que la violencia hacia las mujeres es un grave problema en la salud pública y asimismo violenta los derechos humanos (OMS, 2013). Particular relevancia ha ido cobrando el estudio de aquella que se produce contra la mujer dentro del contexto de las relaciones familiares o de pareja. Diversos estudios a nivel mundial han estimado que entre $10 \%$ y $69 \%$ de las mujeres, de acuerdo al país, refieren haber sido víctimas de agresión física por su pareja (OMS, 2002). A nivel mundial los datos son alarmantes pues alrededor de un tercio (el 30\%) de mujeres han sufrido violencia física y/o sexual cuando mantenían algún tipo de relación sentimental con otra persona. Las cifras alcanzan hasta el 38\% en ciertas regiones. Asimismo el 38\% de la totalidad de homicidios femeninos está ligado a violencia conyugal. El Instituto Nacional de Estadística e Informática (INEI, 2013) refiere que respecto a las mujeres entre los 15 y 49 años: el $35 \%$ han sido víctimas de sus propios esposos o compañeros sentimentales, y el $67.5 \%$ han padecido de "violencia emocional" (o abuso psicológico), violencia que en el extremo lleva al feminicidio, el asesinato de una mujer (OMS, 2013). El Ministerio de la Mujer y Poblaciones Vulnerables considera entre las líneas de investigación que se requieren fortalecer el feminicidio, razón por la cual en un estudio anterior (Quintana, 2015) se constató que los mensajes difundidos en las coplas de carnaval, son una de las formas en las que la cultura inadvertidamente incentiva los estereotipos y actitudes machistas y sexistas, representaciones sociales prevalecientes en los hombres que cometen feminicidio.

\title{
ANTECEDENTES
}

En los últimos tiempos se despliega un gran interés por investigar la problemática de la violencia tanto familiar como sexual en el Perú. Según el Ministerio de la Mujer y Poblaciones Vulnerables (Bardales, 2012), la producción de investigaciones en el rubro oscila en un rango anual de 16 a 26 estudios. En particular se asume una justificación (por parte de los varones) del maltrato hacia la mujer principalmente 
por temas de infidelidad de la pareja o descuido de la progenie. Además, si la pareja rechaza tener relaciones sexuales, toman represalias agresivas y económicas.

Si bien una parte de los hombres que ejercen violencia hacia sus parejas muestran trastornos de la personalidad y/o alto consumo de alcohol u otras drogas (Echeburúa y Redondo, 2010; Bardales, 2012), no se pueden pasar por alto las características del contexto sociocultural que enmarcan la índole de las representaciones sociales, formas de conocer el entorno elaborado y compartido por la sociedad circundante, con prácticos y comunes, (Jodelet,1991). Estas formas de representación se encuentran en constante influencia y transmisión verbal, gráfica e incluso simbólica (Petit y Cilimbini, 2006). Las representaciones adquiridas socialmente conformaran actitudes que en su dimensión cognitiva estereotipa a las personas y objetos sociales. Estos comportamientos son aprendidos en la cultura de referencia, en relación con las representaciones de masculinidad y femineidad socialmente aprobadas. Así, como lo reseñan Larsen y Buss (2005), en todos los estudios sobre estereotipo de género, los hombres son vistos como "más agresivos, autónomos, orientados al logro, dominantes, exhibicionistas y perseverantes", en tanto que las mujeres son vistas como "más afiliativas, deferentes, heterosexuales, de crianza y autodegradantes". De hecho, el subtipo femenino "clásico" incluye amas de casa, secretarias y mujeres maternales, a la vez que "buscadoras de sexo ocasional". Para Glick \& Fiske. (1996) esto sería el sexismo ambivalente, que reconcilia las creencias sexistas hostiles y las benevolentes sin generar sentimientos conflictivos. En el Perú Vargas (2012) coincide haber encontrado en estos estereotipos respecto a los mensajes televisivos referidos a las mujeres y Ardito (2014) referido a otros roles delos ciudadanos.

De otro lado, en la encuesta nacional acerca de la niñez y medios de comunicación (CONCORTV, 2015) se encontró que los niños y adolescentes de 7 a 16 años en un $99 \%$ dicen dedicarse a ver TV de lunes a viernes, eligiendo en un $84 \%$ los programas que ven, siguiendo algunas reglas paternas solo en un $72 \%$, siendo los programas más vistos los Realities de Competencia (Esto es guerra en $50 \%$, Combate en 29\%) y las telenovelas (A1 Fondo Hay Sitio en 36\%), a lo que se añade que en el colegio un 55\% reporta no haber sido orientados acerca de la conveniencia de analizar y comentar lo que ven en la TV. La televisión es observada a diario por $86.8 \%$ de los entrevistados, con una frecuencia de observación de 3 programas diarios a razón de 3 horas. Alrededor del 50\% de niños y adolescentes ven programas de TV mientras se alimenta y tan solo $35 \%$ conversa con sus familiares al alimentarse (CONCORTV, 2014), situación que es mayor en los niveles socioeconómicos C, D y E, al ser esta una de las pocas fuentes de distracción a la que tienen acceso en dependencia a su limitado presupuesto familiar. Al respecto en nuestro medio se han organizado marchas contra la 'TV basura', que es como se ha denominado a los programas televisivos de muy baja calidad educativa o cultural, en los que no se han incluido a las telenovelas (Vivas, 2015). 
Las representaciones sociales suponen la memoria semántica de los individuos y por lo cual una forma adecuada de evaluar las mismas es por medio de la técnica de las Redes Semánticas Naurales (ValdezMedina, 2000).

En consecuencia, es pertinente formular los siguientes problemas:

\section{PROBLEMAS}

- ¿Existirán diferencias significativas en las representaciones acerca de la mujer (mujer-objeto y sexismo ambivalente) entre los grupos de adolescentes que prefieren ver telenovelas y los que prefieren ver realities de competencia?

- ¿Existirán diferencias significativas en las representaciones acerca del hombre entre los grupos de adolescentes que prefieren ver telenovelas y los que prefieren ver realities de competencia?

- ¿Existirán diferencias significativas en las representaciones acerca de la pareja y violencia de pareja entre los grupos de adolescentes que prefieren ver telenovelas y los que prefieren ver realities de competencia?

\section{OBJETIVOS:}

Determinar la diferencia en las representaciones acerca de la mujer (mujerobjeto y sexismo ambivalente) en el grupo de adolescentes que prefieren ver telenovelas, en comparación con las representaciones del grupo de adolescentes que prefieren ver realities de competencia.

Determinar la diferencia en las representaciones acerca de hombre en el grupo de adolescentes que prefieren ver telenovelas, en comparación con las representaciones del grupo de adolescentes que prefieren ver realities de competencia.

Determinar la diferencia en las representaciones acerca de la pareja y violencia de pareja en el grupo de adolescentes que prefieren ver telenovelas, en comparación con las representaciones del grupo de adolescentes que prefieren ver realities de competencia.

\section{HIPÓTESIS}

En ambos grupos de adolescentes se encontraran similares representaciones distorsionadas de la mujer, en tanto mujer objeto y sexismo ambivalente, siendo estos atributos enfatizados más por quienes ven realities de pelea que por quienes ven telenovelas.

En ambos grupos de adolescentes se encontraran similares representaciones distorsionadas del hombre, en tanto machodominante, siendo estos atributos enfatizados más por quienes ven realities de pelea que por quienes ven telenovelas. 
En ambos grupos de adolescentes se encontraran similares representaciones distorsionadas de la pareja, en tanto una relación asimétrica que implica la violencia de pareja, siendo estos atributos enfatizados más por quienes ven realities de pelea que por quienes ven telenovelas.

\section{MÉTODO}

El presente estudio fue realizado a través de una metodología cualitativa, entendiendo que la metodología cualitativa a diferencia de la metodología cuantitativa se orienta por el paradigma inductivo (Quintana, 2006; Mejia,1999), naturalista, de la teoría crítica y el constructivismo (Denman \& Haro, 2000), estableciendo un conjunto de procedimientos que garantizan que los datos recogido construidos y las explicaciones e interpretaciones se aproximen a la realidad (Guba \& Lincoln, 1990), en este estudio particular ello se logrará empleando la técnica de Redes Semánticas Naturales (Figueroa, González y Solís, 1981).

La población objetivo del presente estudio fueron los adolescentes provenientes de los distritos de nivel socioeconómico medio-bajo de Lima metropolitana, con limitado presupuesto familiar que les permite tener como la forma más frecuente de entretenimiento el consumo de programas televisivos.

La muestra estuvo constituida por 500 adolescentes, de ambos sexos, residentes de los conos norte, sur y este de Lima Metropolitana

\section{TÉCNICA O INSTRUMENTO DE RECOLECCIÓN DE DATOS}

Hemos utilizado la técnica de la red semántica, la cual se concibe como un grupo de conceptos elegidos memorísticamente gracias a un proceso reconstructivo, que permite la planeación así como la evaluación subjetiva de acontecimientos, estímulos y conductas. En tal sentido, para dar cuenta de las representaciones sociales acerca de violencia de pareja, estereotipo de género, sexismo ambivalente, elicitadas en la asociación libre que hagan los adolescentes a los conceptos Mujer, Bella, Hombre, Guapo, Conflicto, Violencia y Maltrato, cada uno de estos conceptos, o estímulos instigadores, se presentaran impresos con la siguiente instrucción:

A continuación te mostraremos palabras o frases, frente a cada una de ellas quisiéramos que nos escribas las cinco primeras palabras que se te vengan a la mente, cinco palabras sueltas, que pueden ser verbos, adverbios, adjetivos, sustantivos y nombres, pero no pueden ser artículos ni preposiciones (el, la, los, de, para, pero, por, etc.), no hay respuestas buenas o malas, por favor responde con las cinco primeras palabras que se te ocurra.

Una vez realizada esa tarea se presentara la siguiente instrucción:

Ahora te damos cinco espacios numerados del 1 al 5, para que ordenes las cinco palabras que acabas de escribir de acuerdo con la cercanía o importancia que 
tiene cada una de ellas con la palabra ........... Anota en el espacio correspondiente al número uno la palabra más cercana a ..........., en el dos a la siguiente en cercanía o importancia, y así sucesivamente, hasta anotar las cinco palabras que escribiste.

Para llevar a cabo las asociaciones libres de cada concepto se ofrecerán treinta segundos, así mismo se ofrecerán treinta segundos de tiempo para que jerarquicen cada palabra por orden de importancia o acercamiento con respecto al concepto instigador que indirectamente se le está pidiendo definir.

\section{PROCESAMIENTO DE DATOS}

Después de obtener la información, se calificaron los protocolos de acuerdo a los parámetros de la técnica de Redes Semánticas Naturales y se obtuvieron los valores: Tamaño de la red (TR), núcleo de la red y palabras definidoras (NR), peso semántico (PS) y distancia semántica cuantitativa (DSC).

Para el cálculo se ha seguido el siguiente procedimiento: El tamaño de la red se obtuvó sumando el número total de palabras definidoras frente a cada estimulo instigador producido por los grupos de adolescentes formados según el formato del programa de televisión de señal abierta que más frecuentemente ven (Telenovelas y Realities de Competencia) que mayormente oscilan en un rango de 300 a 500 palabras, mientras que el peso semántico de cada una de las definidoras se obtuvo sumando la ponderación de las frecuencias por la jerarquización asignada por cada grupo de adolescentes, donde la palabra definidora considerada primero en la jerarquía, la palabra definidora considerada más cercana a la palabra estímulo instigador, se multiplica por cinco, la siguiente en la jerarquía por cuatro, la tercera por tres, la cuarta por dos y la quinta y última en la jerarquía de palabras definidoras por uno. El núcleo de la red, está formado por aquellos conceptos que representan (definen) a la palabra estímulo instigadora, considerando las primeras diez palabras definidoras con peso semántico más alto. La distancia semántica se obtiene entre las palabras definidoras del núcleo de la red, asignando a la definidora con peso semántico más alto el $100 \%$ y construyendo los siguientes valores a través de una regla de tres simples a partir de ese valor. Siendo así, la DSC es un indicador de variabilidad intragrupo semántico y el PS es un indicador de la variabilidad intrared semántica.

Para procesar los datos se empleó la hoja de cálculo Excel y el SPSS v.20, estableciendo finalmente tablas y gráficos. La interpretación de los citados resultados se realizó mediante la técnica de análisis de contenido y elementos de la teoría fundamentada (Álvarez Gayou, 2003).

\section{RESULTADOS}

Como se puede apreciar en la figura 1, los hombres en general tienen más definidos los cinco principales términos definitorios de MUJER si bien con independencia 
del termino bella que es compartido por todos los sujetos de la muestra se encuentran semánticamente más alejados del núcleo ( $60 \%$ a más), que en el caso de las mujeres, (55\% a menos), estas últimas por el contrario solo mantienen los tres primeros rasgos definitorios con variada distancia semántica y ya no presentan en el caso de las que ven telenovelas, o los presentan dramáticamente alejados semánticamente, en el caso de las que ven realities de competencia ( $70 \%$ a más), los términos definitorios linda e inteligente, lo cual estaría indicando que este estereotipo estaría consolidado en las mujeres, mientras que corresponde solo parcialmente en los varones.

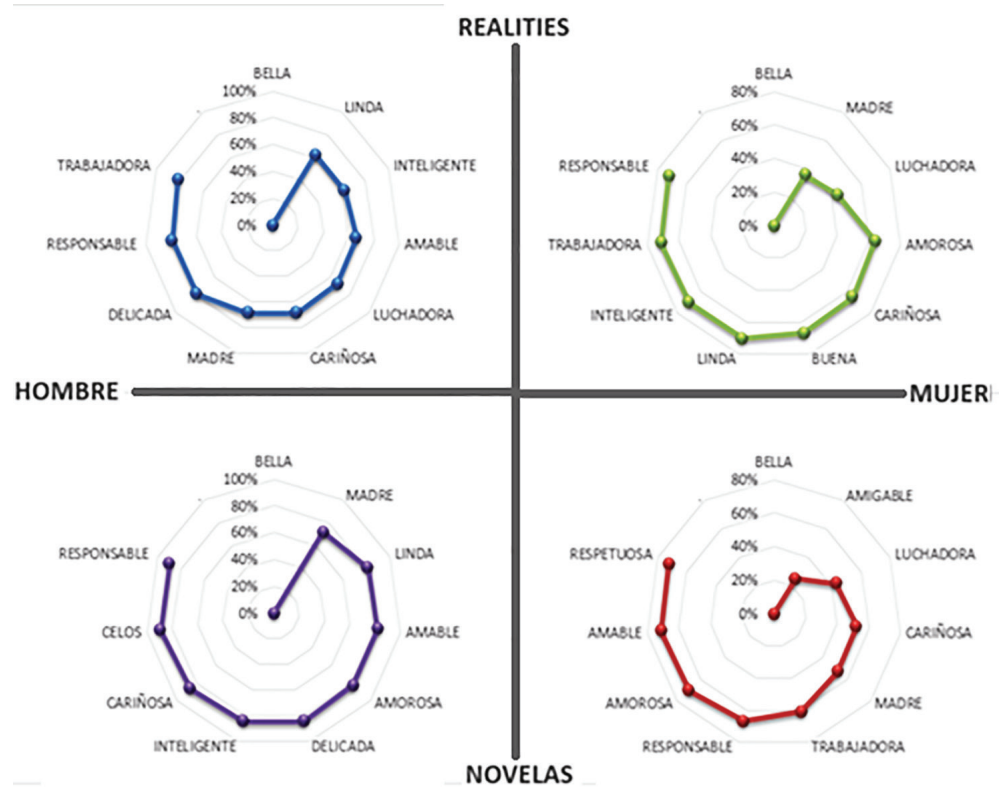

Figura 1. Representaciones acerca de la mujer entre los grupos de adolescentes que prefieren ver telenovelas y los que prefieren ver realities de competencia.

Específicamente los hombres que ven realities de competencias, frente a la palabra estímulo MUJER, destacan, en un mayor porcentaje, la belleza de la mujer en el aspecto físico, en tanto que es reforzada por la cualidad física de ser linda, con rasgos de inteligencia ( $60 \%$ a menos). Mientras que la opinión más alejada del núcleo semántico de este grupo de hombres, destacan las cualidades de la mujer como la de ser amable, luchadora, cariñosa, maternal, delicada, responsable y trabajadora ( $80 \%$ a más). Asimismo la opinión de las mujeres que ven realities de competencias, frente a la palabra estímulo MUJER, en primer lugar también es bella, pero semánticamente bastante cercanos también destacan los atributos de madre y luchadora ( $45 \%$ a menos). En cuanto a la opinión de los hombres que ven telenovelas más cercanos al núcleo semántico destacan la belleza de la mujer (bella, linda), coincidiendo con el estereotipo de la mujer en publicidad, donde se la describe 
más por su apariencia que por sus capacidades y la cualidad maternal ( $75 \%$ a menos) (Bernad, E., 2010, Amaya, S., 2004), mientras que las mujeres que ven telenovelas representan, semánticamente más alejadas, las cualidades de la mujer como la de ser maternal, luchadora, cariñosa, responsable y trabajadora ( $75 \%$ a más), vale decir el estereotipo tradicional de la mujer, como la responsable del hogar y de la crianza de los hijos. Esto es reforzado por las respuestas al estímulo Bella.

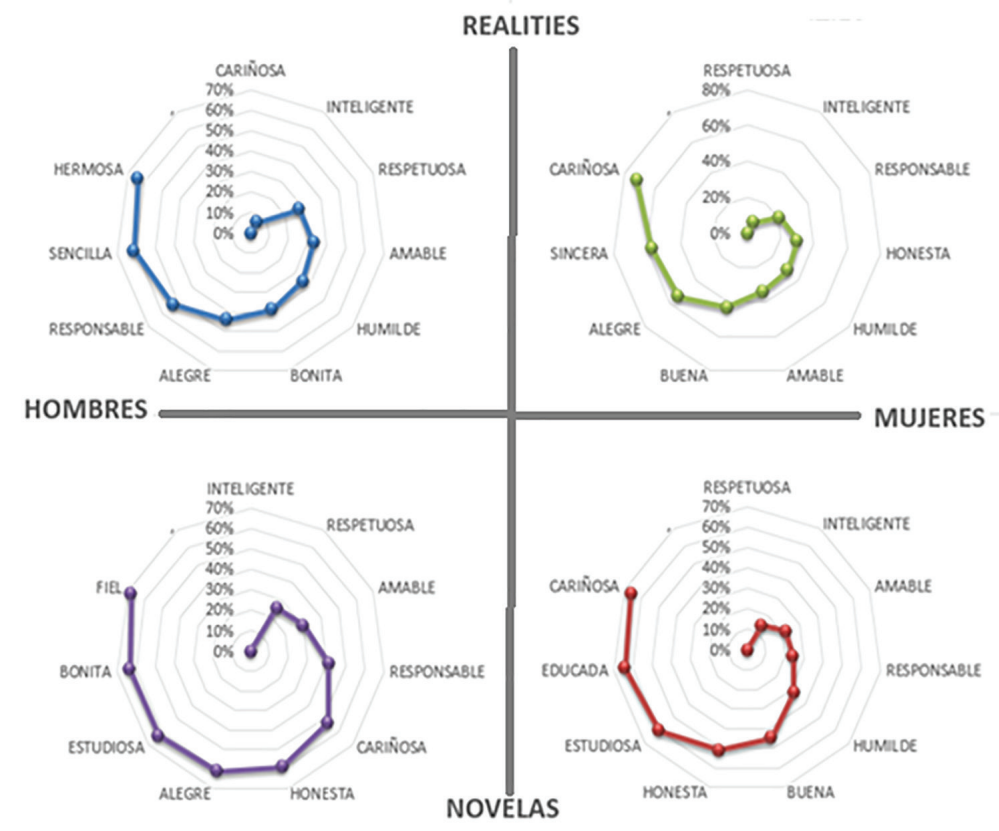

Figura 2. Representaciones acerca bella entre los grupos de adolescentes que prefieren ver telenovelas y los que prefieren ver realities de competencia.

En la figura $2 \mathrm{Al}$ igual que frente al estímulo anterior las mujeres en general tienen más definidas las cinco principales palabras definitorias de BELLA (20\% a menos) si bien con independencia del termino inteligente que es compartido por todos los sujetos de la muestra (15\% a menos), por el contrario los varones se encuentran semánticamente más alejados del núcleo (30\% a más) que en el caso de las mujeres, ellos solo mantienen las tres primeras palabras definitorias con variada distancia semántica (35\% a menos) y presentan dramáticamente alejadas semánticamente las palabras definitorias responsable (más de 45\%) y alegre (más de 55\%), lo cual estaría indicando que este estereotipo estaría consolidado en las mujeres, mientras que corresponde solo parcialmente a los varones. podemos observar que tanto hombres como mujeres independientemente de la preferencia televisiva presentan como palabras definidoras más cercanas al núcleo semántico del estímulo BELLA las palabras respetuosa, inteligente, responsable y amable (menos 
del 45\%), mostrando un estereotipo donde la belleza de la mujer es asociada a la vez a la inteligencia, contraviniendo los estereotipos tradicionales de la publicidad, en tanto mujer destacable por sus atributos físicos y el de la mujer concebida en el imaginario popular como respetuosa, responsable y amable, es decir subordinada al varón, con limitada inteligencia. Estereotipo emergente de la mujer en el que si bien se le adjudica la cualidad de inteligente no se deja de destacar en primer lugar su subordinación como característica deseable y definitoria de la mujer.

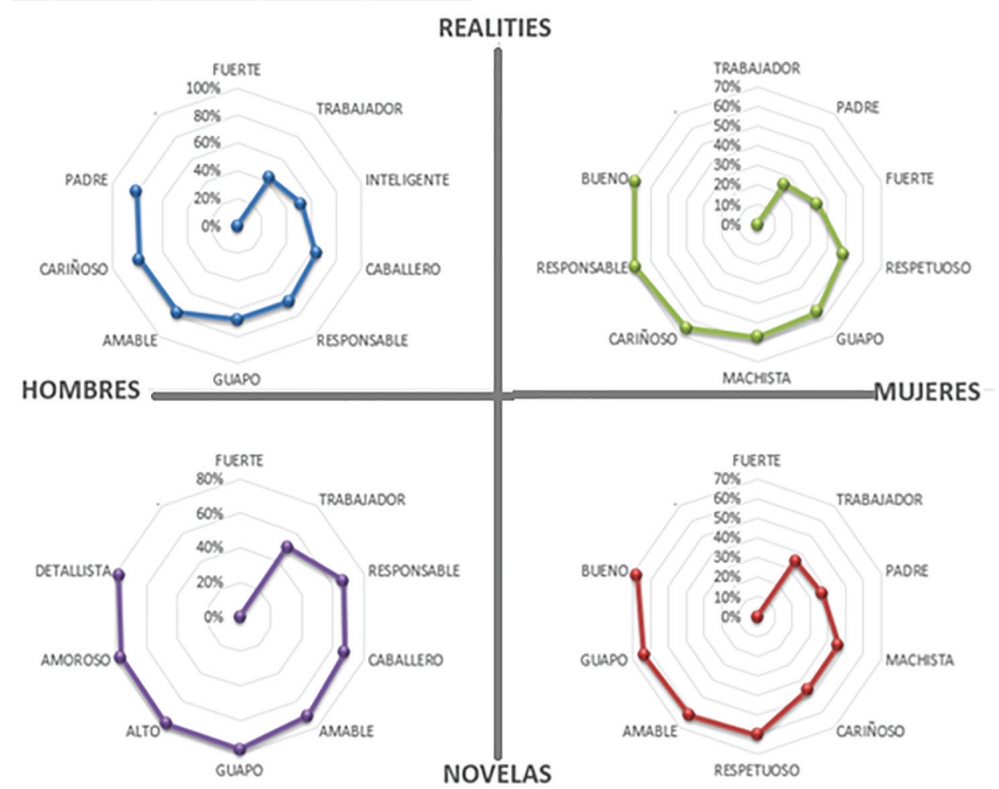

Figura 3. Representaciones acerca del hombre entre los grupos de adolescentes que prefieren ver telenovelas y los que prefieren ver realities de competencia.

Respecto al estereotipo de HOMBRE nuevamente se observa que los hombres en general tienen más definidos cuatro de los cinco principales términos definitorios y si bien con independencia del termino fuerte que es compartido por todos los sujetos de la muestra a excepción de las mujeres que ven realities de competencia (para las que el núcleo lo representa el termino trabajador), se encuentran semánticamente más alejados del núcleo trabajador, responsable y caballero ( $40 \%$ a más) que en el caso de las mujeres, estas últimas por el contrario solo mantienen los tres primeros rasgos definitorios con variada distancia semántica Fuerte, trabajador y padre $(40 \%$ a menos), y ya no presentan en el caso de las que ven telenovelas o lo presentan dramáticamente alejado semánticamente en el caso de las que ven realities de competencia ( $70 \%$ ) el término definitorio responsable, lo cual estaría indicando que el primer estereotipo del hombre como protector y caballero, propio del sexismo ambivalente, estaría parcialmente consolidado en los varones, mientras que el último 
estereotipo, del hombre en tanto fuerte y proveedor de recursos, correspondería a las mujeres, el cual no deja de coincidir con el estereotipo cultural, que ha legitimado históricamente el poder y la dominación del hombre sobre la mujer, sea por la fuerza física o por ser proveedor de recursos, promoviendo la dependencia y subordinacion de la mujer (Ibáñez, 2004).

Concretando, en toda la muestra coincide en considerar como cualidades primordiales del concepto HOMBRE, el ser fuerte y trabajador. Las cualidades que se presentan más alejadas del núcleo semántico en estos cuatro grupos, son Guapo, cariñoso y responsable (50\% a más), entre otras.

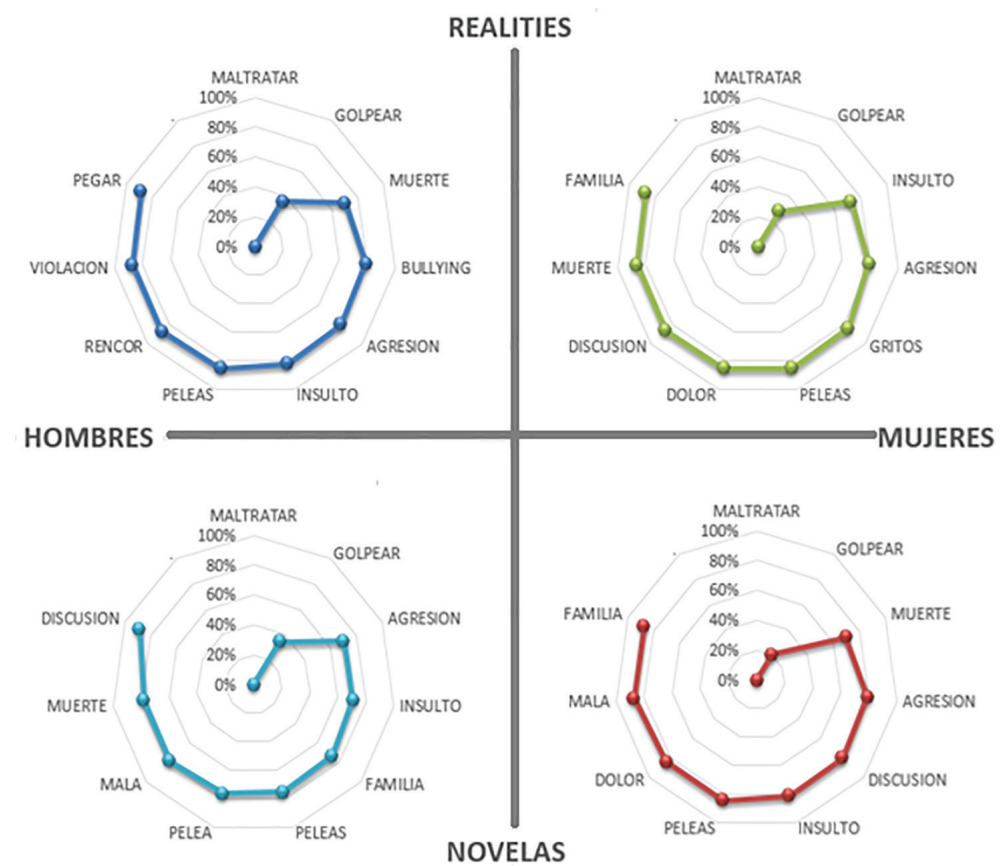

Figura 4. Representaciones acerca de violencia entre los grupos de adolescentes que prefieren ver telenovelas y los que prefieren ver realities de competencia.

En la figura 4 podemos observar que el estereotipo de violencia es consistente en prácticamente todos los integrantes de la muestra. Así todos los grupos coinciden en destacar como semánticamente nucleares las palabras definitorias Maltratar y Golpear ( $40 \%$ a menos) en los varones y (25\% a menos) en las mujeres, en ese orden, acompañadas aunque con menor peso semántico (70\% a más) de otras como: insulto, agresión y muerte. Quedando con mucho menor peso semántico definidoras más simétricas de violencia como peleas o discusión $(80 \%$ a más). Concepción de la asimetría en la violencia que asociada a las definitorias del varón como fuerte y dominante, indicaría una relación asimétrica que implica la violencia de pareja, que en su imaginario puede llegar al homicidio, como lo 
evidencian al incluir el concepto muerte en sus definitorias de violencia, todos los integrantes de la muestra.

\section{DISCUSIÓN}

Para las adolescentes mujeres de la muestra los significados psicológicos inferidos de las redes semánticas formadas ante las palabras estímulos hombre, mujer, que como se ha dicho líneas atrás son bastante estables semánticamente, nos remite a los roles de género tradicionales que se instauran dentro de un marco de relación asimétrica, en la que los hombres y "lo masculino" ocupan la posición dominante, como refiere Janos, Espinosa (2014); todo ello enmarcado en un sistema de organización social patriarcal, evidenciándose una aceptación mayoritaria de la autoridad de la figura masculina proveedora, productiva (Connell, 1995); siendo las palabras definidoras que lo delinean fuerza y trabajador.

Además, para las adolescentes las palabras definidoras asociadas a MUJER son bella y madre. Ésta última enfatiza el rol materno de la figura femenina, decisorio para su realización como mujer (IOP, 2014), y la primera definitoria, la belleza que en los adolescentes se constituye en un estereotipo al ligarse al culto de la corporalidad, reforzada mediante la afirmación de la moda o a la expresión de la desnudez como manifiesta Aguirre (1994), aunado a un sistema social que prioriza el que las mujeres sean principalmente valoradas por su lo físico antes que por su intelectualidad (Artigas, 2007) . Al respecto la televisión hace uso del carácter evocador del estereotipo para elaborar discursos visuales y verbales de fácil identificación con el público adolescente, naturalizando y reforzando a su vez el mantenimiento de estos estereotipos. Es menester señalar el estereotipo emergente de la mujer, presente en los adolescentes de la muestra a excepción de las mujeres que ven telenovelas, en el que si bien se le adjudica la cualidad de inteligente, no se deja de destacar en primer lugar su subordinación como característica deseable y definitoria de la mujer.

Representaciones similares, y semánticamente estables, con respecto a la violencia que se puede suscitar en una relación de pareja se dieron para todos los adolescentes de la muestra, las palabras definidoras maltratar, golpear, agresión, peleas, insulto nos permiten inferir sobre el conocimiento de la multiplicidad de manifestaciones de la violencia de orden físico y psicológico, evidenciándose uno de los mayores valores del índice J para esta palabra estímulo, en este estudio. También cabe indicar que aun cuando presentan mayor distancia semántica las palabras definidoras gritos, dolor, violación ( $80 \%$ a más), nos permiten ampliar nuestro acceso al imaginario de estos adolescentes con respecto a la relación asimétrica de dominancia y subordinación que implica la violencia de pareja. Y explicitar como los estereotipos de género dan más fuerza al supuesto "derecho del marido" para tener control de su pareja incluso ejerciendo violencia en ella" (Morrison, Buvinic, \& Shifter, 2005). 
Es importante señalar que en los adolescentes una de las palabras definidoras alarmante es muerte que tiene un orden jerárquico mayor en el grupo de adolescentes varones que ven realities de competencia y mujeres que ven telenovelas $(65 \%$ a menos). Lo cual nos lleva a cuestionarnos si esta palabra definidora se constituye en el imaginario de estos jóvenes en una alerta de cómo la violencia de pareja puede conducir al feminicidio.

\section{CONCLUSIONES}

- Las adolescentes mujeres limeños tienen una representación semánticamente más estable de los roles de género tradicional, de una relación asimétrica de predominio masculino.

- Para estas adolescentes las palabras definidoras más cercanas al núcleo semántico del estereotipo mujer son bella y madre, enfatizándose la belleza y el rol materno de la figura femenina,

- Se observan representaciones similares en todos los integrantes de la muestra con respecto a la violencia en términos de abuso que se puede suscitar en una relación de pareja.

- Todos los adolescentes participantes son conscientes de la multiplicidad de manifestaciones de la violencia de orden físico y psicológico.

\section{RECOMENDACIONES}

A partir de los resultados obtenidos creemos que es necesario plantear una propuesta estratégica promocional y preventiva que fomente una cultura de paz con equidad en la cual las mujeres y los hombres sean asumidos como semejantes y como personas sujetas a derechos y libertades en igualdad de condiciones.

Por ende es fundamental que se transformen y/o se generen nuevos escenarios en el ámbito mediático en que se favorezca la propagación de representaciones femeninas distanciadas de patrones sexistas ambivalentes y más próximas a la concepción de las mujeres con capacidad de agencia, como agente activo de cambio en relación a los diferentes roles que protagoniza en la sociedad y no caer en una polarización en que la mujer que demuestra fortaleza, liderazgo se convierte en "el nuevo hombre", evidenciándose el predominio de un imaginario masculino; pero tampoco como justificación para contraponerse a las creencias en que se visualizan a las mujeres como manipuladoras que utilizan su atractivo sexual para dominar a los hombres, como refieren Glick.\& Fiske (1996) sobre el sexismo hostil. 


\section{REFERENCIAS BIBLIOGRAFICAS}

Aguirre, A (1994) Psicología de la adolescencia. España: Ed. Boixareau Universitaria.

ÁlvarezGayou, J. L. (2003). Cómo hacer investigación cualitativa. Fundamentos y metodología. México: Paidos.

Amaya, S., (2004) La represión sexual de la mujer en la publicidad.Etic@net. 2(3). 1-8. Visto el 15/03/2016 en http://www.ugr.es/ sevimeco/revistaeticanet/Numero3/

Ardito (2014) Discriminación y programas de televisión. Consultoría sobre estereotipos $y$ discriminación en la televisión peruana. Lima: CONCORTV.

Artigas, M (2007) Los medios y el prototipo de belleza en la sociedad actual. V Congreso Virtual "La tesis en comunicación. Centralidad de los antecedentes y el estado del arte en la elaboración del Plan de Tesis".

Bardales, O. (2012). Estado de las investigaciones sobre violencia familiar y sexual 20062010. Lima: Ministerio de la Mujer y Poblaciones Vulnerables.

Bernad, E. (2010): Ilicitud de las representaciones degradantes y humillantes del cuerpo femenino en la publicidad. Especial referencia a la anorexia. Icono14, 8 (3), 186207.

CONCORTV, (2014) Estudio cuantitativo sobre consumo televisivo y radial en niños, niñas y adolescentes informe final. Visto el 09712/2015 en: http://www.concortv.gob. pe/index.php/investigaciones/encuestas.html.

CONCORTV, (2015) Estudio sobre consumo radial y televisivo. Visto el 09712/2015 en http://www.concortv.gob.pe/index.php/investigaciones/encuestas.html.

Connel, R. (1995) “The Social Organization of Masculinity” de Masculinities, University of California Press, Berkeley.

Denman, C., \& Haro, J. A. (2000). Por los rincones. Antología de métodos cualitativos en la investigación social. Hermosillo: El Colegio de Sonora.

Echeburúa, E. y Redondo, S. (2010). ¿Por qué víctima es femenino y agresor masculino? La violencia contra la pareja y las agresiones sexuales. Madrid: Pirámide.

Figueroa, J., González, E., y Solís, V.; (1981) Una aproximación al problema del significado: las redes semánticas. Revista Latinoamericana de Psicología. 13,3, 447458.

Glick, P. \& Fiske, F. (1996) The Ambivalent Sexism Inventory: Differentiating Hostile and Benevolent Sexism. Journal of Personality and Social Psychology y the American Psychological Association Vol. 70, No. 3, 491-512.

Guba, E., \& Lincoln, Y. (1990). Fourth generation evaluation. London: Sage.

Ibáñez, M. (2004). Violencia doméstica en Euskadi: malos tratos a la mujer: Análisis definición del proceso de ruptura con una relación de maltrato. Vitoria-Gasteiz: Servicio Central de Publicaciones del Gobierno Vasco.

Instituto Nacional de Estadística e Informática (2013). Violencia de Género. Visto el 09712/2015 en https://www.inei.gob.pe/estadisticas/indicetematico/ violenciadegenero7921/. 
Janos, E. y Espinosa, A (2014) Representaciones sociales sobre roles de género y su relación con la aceptación de mitos y creencias sobre la violencia sexual. Límite. Revista Interdisciplinaria de Filosofía y Psicología Volumen 10, № 33, pp. 5-15. Lima- Perú.

Jodelet, D. (1991) Representaciones sociales. Un área en expansión en: Páez, D., San Juan, C., Romo, 1., Vergara, A., SIDA, imagen y prevención, Ed. Fundamentos, Madrid.

IOP PUCP. (2014) Estudio Familia, Roles de Género y Violencia de Género. Lima: (Informes de Estudio, $\mathrm{N}^{\circ} 2$ )

Larsen, R.J. y Buss, D.M. (2005). Psicología de la personalidad. Dominios de conocimiento sobre la naturaleza humana. 2da. Ed. México: McGrawHill.

Mejia, J. (1999). De la construcción del Conocimiento Social a la Práctica de la Investigación Cualitativa. Revista Investigaciones sociales 2 (3). IHSUNMSM.

Morrison, A., Buvinic, M., \& Shifter, M. (2005). América violenta: factores de riesgo, consecuencias e implicancias para las políticas sobre la violencia social y doméstica. En Frhling, . S. Tulchin, . A. Olding, E. Imerman, . Escobar, Crimen y violencia en América Latina seguridad ciudadana, democracia y estado. Bogotá; México: Fondo de Cultura Económica.

Moscovici, S. (1976) Pensamiento y vida social, Paidós, Barcelona.

Organización Mundial de la Salud. (2002) Informe mundial sobre la violencia y la salud: resumen. Washington, D.C.: Autor

Organización Mundial de la Salud. (2013) Comprender y abordar la violencia contra las mujeres. Visto el 09/12/2015 en http://www.who.int/reproductivehealth/topics/ violence/vaw_series/es/.

Petit y Cilimbini (2006) Representaciones Sociales y Medios de Comunicación. Visto el 09/12/2015 en http://www.ocw.unc.edu.ar/facultaddepsicologia/ psicologiadelasmasasylosmediosde/actividadesymateriales/2008/ articulorepresentacionessocialesymediosde/view

Quintana, A. (2006) Metodología de Investigación Científica Cualitativa. En Quintana, A. y Montgomery, W. (Eds. Psicología: Tópicos de actualidad. Lima: UNMSM.

Quintana, A. (2015) Sexismo, misoginia y estereotipos de la mujer y de género en las coplas del carnaval de Cajamarca. Inédito. Articulo aceptado para ser publicado en la Revista de Psicología UCV, Lima.

Valdez Medina, J. L. (2000) Las redes semánticas naturales usos y aplicaciones en psicología social. Universidad Autónoma del Estado de México. México. Maz Ediciones.

Vargas (2012) Análisis de la imagen de las mujeres en la programación televisiva transmitida en Lima. Lima: CONCORTV.

Vivas (2015) Todo lo que necesitas saber sobre la televisión basura. Diario el Comercio del Domingo 22 de febrero del 2015. 